\title{
ECRH scenarios with selective heating of trapped/passing electrons in the W7-X Stellarator
}

\author{
N.B. Marushchenko ${ }^{1}$, C.D. Beidler ${ }^{1}$, V. Erckmann¹, J. Geiger ${ }^{1}$, P. Helander ${ }^{1}$, H.P. Laqua ${ }^{1}$, \\ H. Maassberg ${ }^{1}$, and Y. Turkin ${ }^{1}$ \\ ${ }^{1}$ Max Planck Institute for Plasma Physics, Wendelsteinstr. 1, D-17491 Greifswald, Germany
}

\begin{abstract}
Using specific features of the magnetic equilibrium in the W7-X stellarator, the ECRH scenarios with combined X2 and X3 modes are discussed. The RF beams for operation with X2 and X3 modes need to be launched from low- and, via the remote steering launcher, high-field-side, respectivaly, in the different crosssections of the device where the maximum and minimum of the magnetic field located. The aim is to explore the possibility of selective heating of the different classes of electrons, passing and trapped, by changing direction of the beam for X3 or switching between the beams for X2 and X3 launched from the different ports. The numerical predictions for this kind of experiments in $\mathrm{W} 7-\mathrm{X}$ are performed by coupled transport and ray tracing codes.
\end{abstract}

\section{Introduction}

The main scientific goal of the Wendelstein 7-X stellarator (W7-X) $[1,2]$ is to demonstrate good plasma confinement with steady-state operation and reactor-relevant parameters. The system for electron cyclotron resonance heating (ECRH) in W7-X has been designed for continuous operation with total injected power up to $10 \mathrm{MW}$. The ECRH system contains ten gyrotrons, which generate waves at $140 \mathrm{GHz}$ with a power up to $1 \mathrm{MW}$ or (optionally) at $105 \mathrm{GHz}$ with $0.5 \mathrm{MW}$ each, respectively $[3,4]$. The main ECRH scenarios are designed for operation at $B_{0} \simeq 2.5 \mathrm{~T}$ and $140 \mathrm{GHz}$ with the X2-mode $\left(n_{e}<1.2 \times 10^{20} \mathrm{~m}^{-3}\right)$ or O2-mode $\left(n_{e}<2.4 \times 10^{20} \mathrm{~m}^{-3}\right)$. The main launch-ports are situated near the "bean-shaped" poloidal plane, where the maximum of $B$ is located and the radial dependence of $B$ is tokamak-like. Launching of the power from lowfield-side (LFS) through these ports minimizes direct heating of the trapped electrons for both X2 and O2-modes. Additionally, the RF power can to be injected using the "remote steering launcher" (RSL) [5, 6] which allows for rapid changing the beam directions in limits $\pm 15^{\circ}$ with respect to the central line of RSL that corresponds to changes of $N_{\|}$near the axis from 0.4 to 0.8 (the central line, marked here as " 0 deg" corresponds to $N_{\|} \simeq 0.6$ ), and this opens the new possibilities for more complex experiments.

Due to rapid redistribution over the flux-surface, the power density absorbed by passing electrons is sufficiently low in comparison with the rate of thermalization, $P_{\text {abs }}^{\text {ass }} \ll$ $n_{e} T_{e} v_{e}$, that makes linear treatment to be appropriated. Usually, similar argumentation can be applied also for trapped electrons and this is the basic assumption for the transport theory. However, there is a number of experiments (see for example [7-11]) as well as theoretical in- vestigations $[8-10,12,13]$ which indicate significant kinetic effects for the transport in the case when a substantial fraction of the RF power is absorbed by trapped electrons, $P_{\text {abs }}^{\text {trap }}>P_{\text {abs }}^{\text {pass }}$. This can be especially important for stellarators, where the trapped electrons, being locked within the toroidal ripples, do not spread the absorbed power toroidally over the magnetic surface, and in the poloidal direction only do so by their precessional motion. Then, the power absorbed by trapped electrons can be thermalized only by collisions with passing electrons. In this case, the local absorbed power density in the heated ripple can be significantly increased, $\left(d P_{\text {abs }}^{\text {trap }} / d V\right)_{\text {local }}>d P_{\text {abs }} / d V$ and, in some cases, quasilinear or even non-linear [14] effects may be important (here, the left part of inequality corresponds to the "local" density of absorbed power in the ripple where the power is absorbed, and the right part to the power averaged over the entire magnetic surface). As a consequence, the generation of additional convective losses [12] can be observed. These features are still inadequately explored and both experimental and theoretical researches in this direction are desirable.

In order to study kinetic effects induced by the direct heating of trapped electrons in W7-X, the scenarios with high-field-side (HFS) launching near the minimum of $B$ can be applied since those can potentially produce the effects not accounted in the standard neoclassical theory. For this goal, two additional ports (named as N-ports) near the "triangular" poloidal plane have been reserved. Here, the minimum of $B$ is located and, in contrast to the "beanshaped" plane, the radial gradient of $B$ is small and can even change sign along the sight-line. Throught the Nports, the RF power has to be injected via RSL $[5,6]$. Using the features of the N-ports and a unique flexibility of 
W7-X magnetic configuration, the conditions for significant and even dominating contribution of trapped electrons in cyclotron absorption can be found.

The W7-X magnetic configuration (a low-shear configuration of the Helias type with five field periods) is quite flexible and well optimized with respect to low neoclassical transport and good confinement of fast particles. For a choice from the configuration space, there are several options: the "standard" magnetic configuration, the "lowmirrors" and the "high-mirrors" configurations with the various ratio $B_{01} / B_{00}$ (here, $B_{n m}$ is the Fourier harmonic with $n$ as poloidal and $m$ as toroidal number). While for the "standard" magnetic configuration an operation with both the 2nd and 3rd harmonics is possible only using the different frequencies $(105 \mathrm{GHz}$ for $\mathrm{X} 2$ and $140 \mathrm{GHz}$ for $\mathrm{X} 3$ ) and reduced magnetic field, $B_{00}=1.69 \mathrm{~T}$, for the "high-mirror" configuration with sufficiently large mirrorratio the heating of both $\mathrm{X} 2$ and $\mathrm{X} 3$ at the same frequency is possible for the nominal magnetic field about $2.5 \mathrm{~T}$.

The present work is devoted to study of two ECRH scenarios with changing the fraction of trapped electrons responsible for the absorption. The first one, with the "standard" magnetic configuration, $B_{01} / B_{00}=0.043$, is the operation with the RF beams of $105 \mathrm{GHz}$ (X2-mode) and $140 \mathrm{GHz}$ (X3-mode) injected from the different ports. In the second scenario, with the "high-mirror" configuration, $B_{01} / B_{00}=0.246$, both X2 and X3 heating are produced at the same frequency, $140 \mathrm{GHz}$, for the beams launched from the different ports (for X2 from the main ports located near the "bean-shaped" plane, and X3 from the N-ports near the "triangular" plane). While the X2mode is absorbed near the maximum of $B$ only by passing electrons, the power fraction from the $\mathrm{X} 3$-mode absorbed near the minimum of $B$ by trapped electrons can be varied practically from zero to almost complete absorption.

Simulations were performed with help of the following numerical tools: 3D ray-tracing code TRAVIS [15] coupled self-consistently with the 1D-transport code NTSS [16], which is based on comprehensive neoclassical and simple turbulent transport models. The magnetic equilibriums with finite- $\beta$ effects accounted were precalculated by the VMEC code [17] as well as the transport coefficients calculated by the DKES code [18]. The present understanding and all theoretical assumptions that adopted for modeling are based on the substantial experience from W7-AS and other experiments $[8,9,11,19]$.

\section{LFS X2 + HFS X3 with steering via RSL in "standard" configuration}

The first example of selective heating of the trapped electrons is based on the combined LFS X2 and HFS X3 scenario with rapid changing via RSL the direction of the $\mathrm{X} 3$ beam and, respectively, significantly changed the fraction of power absorbed by trapped electrons.

In the simulations, eight $105 \mathrm{GHz}$ beams of X2-mode with 0.5 MW each are assumed to be launched from LFS close to the "bean-shaped" plane. For illustration, one X2-mode beam is shown in Fig. 1 (top). The RF power
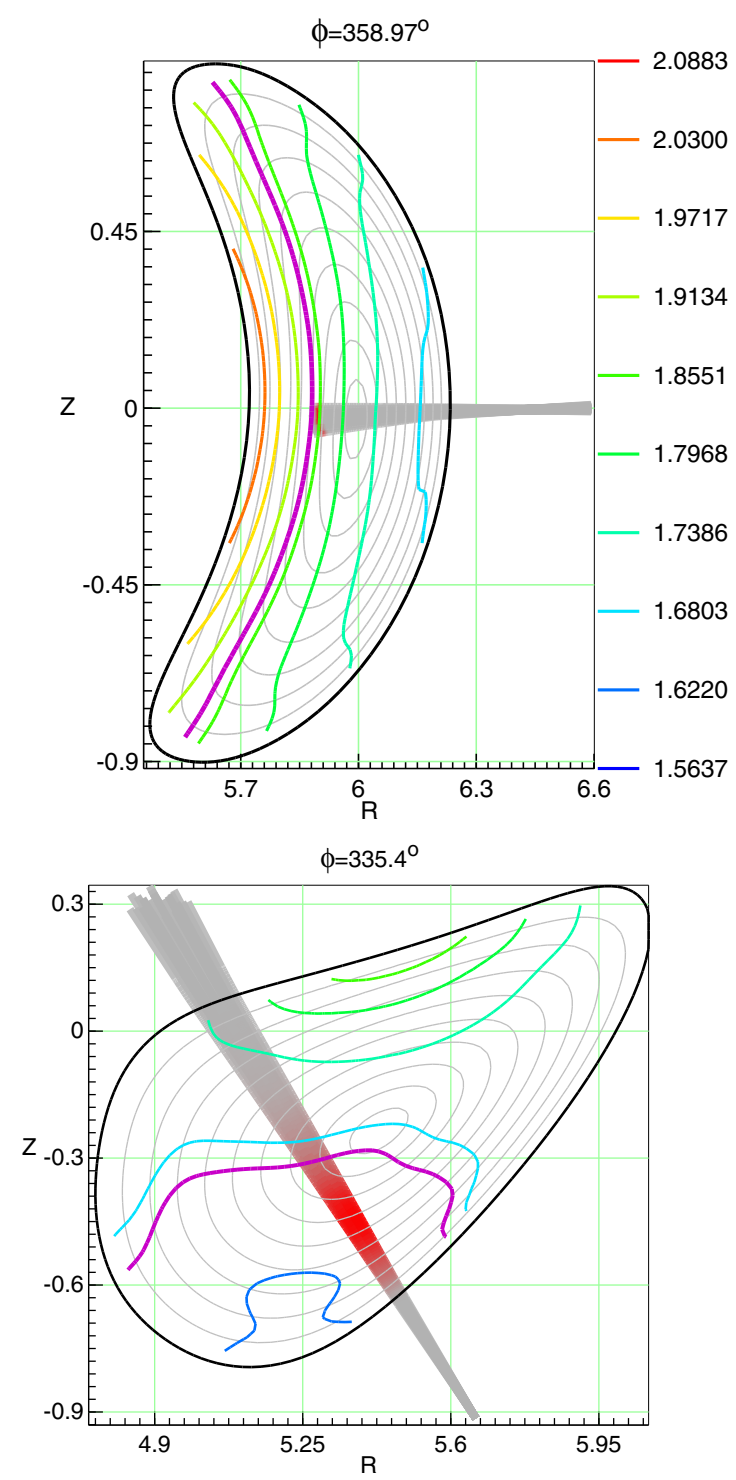

Figure 1. Projections of the different RF beams onto the RZ cross-sections are shown. The toroidal angle of the cross-section corresponds to where the deposition is maximal. $B$-isolines together with the resonance value (magenta) are shown. The absorption is shown in red. Top: the beam at $105 \mathrm{GHz}$ (X2-mode) launched from LFS close to the "bean-shaped" plane (main port near the maximum of $B$ ). In the absorption zone $N_{\|} \simeq-0.25$. Bottom: the beam at $140 \mathrm{GHz}$ (X3-mode) launched from HFS near the "triangular" plane (N-port near the minimum of $B$ ). In absorption zone $N_{\|} \simeq 0$.

is deposited in the central region but off-axis, since the resonance magnetic field is shifted to the high-field side. The obliqueness is not very large (in the absorption zone $N_{\|} \simeq-0.25$ ) and the Doppler shift is not significant. All the X2-beams have practically the same deposition profiles with complete single-pass absorption (total power $4 \mathrm{MW}$ ). The angles for co- and counter-directed beams are chosen in such a way that the residual current drive would be sufficient for compensation of the bootstrap current.

Two RF beams in X3-mode (140 GHz) with $1 \mathrm{MW}$ each are launched from HFS near the "triangular plane" using the "remote steering launcher" (RSL) $[5,6]$, and direction of the beams can be changed practically immedi- 

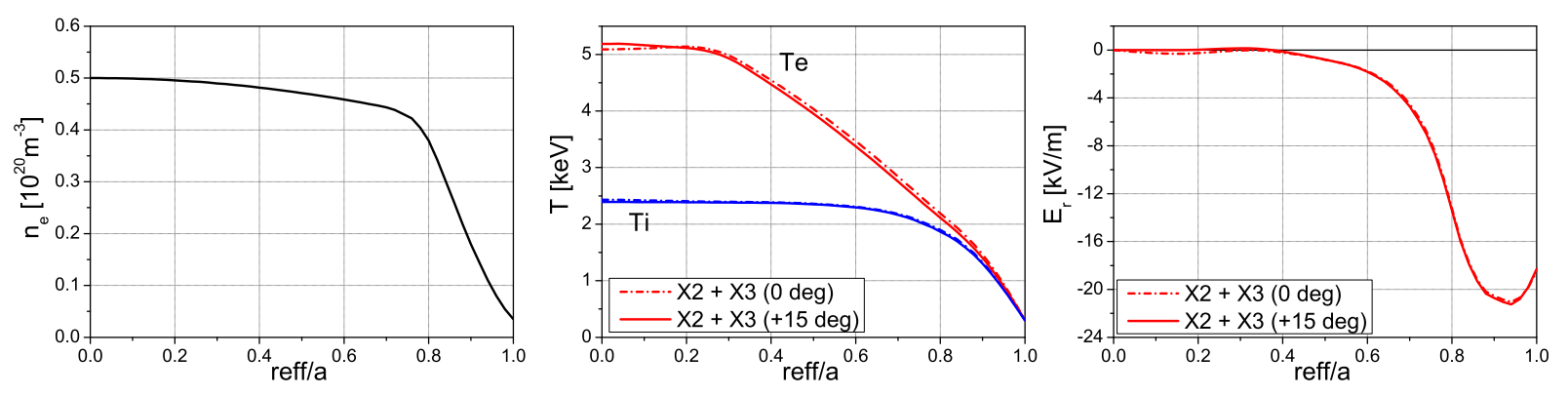

Figure 2. Plasma profiles established for combined LFS X2 + HFS X3 heating with $P_{R F}^{\text {total }}=6 \mathrm{MW}$ in "standard" magnetic configuration are shown. The results for " 0 deg" direction of X3 is shown by dot-dashed lines and for " +15 deg" by solid lines. Left: density (fixed in simulations, assumed to be controlled). Middle: electron and ion temperature profiles for both cases, " 0 deg" and "+ 15 deg". Right: radial electric field for both cases.

ately in the limits \pm 15 degree from the central line of the launcher. While the angle "- $15 \mathrm{deg}$ " is far from the optimal due to strong refraction $\left(N_{\|} \simeq 0.8\right)$, here, only two directions, marked as " 0 deg" and " $+15 \mathrm{deg}$ ", are considered (in Fig. 1 (bottom), the beam for " 0 deg" is shown). Both these angles correspond to rather high obliqueness, with $N_{\|} \simeq 0.5-0.6$ and $N_{\|} \simeq 0.4-0.5$ in absorption zone, respectively. For the X3-mode plasma is optically gray, but the single-pass absorption is high enough, varying from $85 \%$ for " 0 deg" to $98 \%$ for " +15 deg".

In the simulations, neoclassical core confinement with empirical anomalous transport at the plasma edge is assumed. The anomalous diffusivity is taken to scale inversely with the plasma density in the region of high density gradient and to decay exponentially towards the plasma axis. In the transport code, the radial electric field, electron and ion temperatures are calculated selfconsistently with the power deposition profiles calculated by the ray-tracing code. The shape of the density profile, shown in Fig. 2 (left), with a gradient region of about $10 \mathrm{~cm}$ is fixed and $n_{e}(0)=0.5 \times 10^{20} \mathrm{~m}^{-3}$ (the cut-off for $\mathrm{X} 2$-mode at the frequency $105 \mathrm{GHz}$ is $0.68 \times 10^{20} \mathrm{~m}^{-3}$ ). For this density plasmas with $T_{e}>1 \mathrm{keV}$ are optically thick for X2 with highly localized deposition profiles. The temperature profiles predicted by transport simulations (Fig. 2 (middle)) are flat within the normalized radius $\rho<0.28$, with $T_{e}(0) \simeq 5.1 \mathrm{keV}$ and $T_{i}(0) \simeq 2.4 \mathrm{keV}$. In Fig. 2 (right) also the radial electric field is shown. One can see that $E_{r}$ is very small within the heated region. The most important is that the predicted profiles are practically the same for both checked cases which correspond to the different directions of the $\mathrm{X} 3$ beams.

For comparison, direction of the beam for HFS X3mode is changed to be along the " 0 deg" line. Since the deposition profile of the $4 \mathrm{MW}, \mathrm{X} 2$-mode contribution hardly changes, the temperature profiles predicted by the transport code are practically the same, the deposition of $2 \mathrm{MW}$ from the X3-mode is shifted toward the axis; see Fig. 3 (c). However, the main effect is the following: trapped electrons absorb in this case approximately $94 \%$ from the $\mathrm{X} 3$-mode, that is about $28 \%$ of the total absorbed power; see Fig. 3 (d).
Since the fraction of power absorbed by trapped electrons from the HFS X3 mode injected near the "triangular" plane is sensitive to the location of the resonance, it is also sensitive to the plasma $\beta$-value. The modeling is performed for the "standard" magnetic configuration with the parabolic $\beta$-profile and $\langle\beta\rangle=2 \%$ (this value is consistent with the transport calculations which give $\langle\beta\rangle \simeq 2.5 \%$ ). The magnetic field with $B_{00}(0)=1.693 \mathrm{~T}$ guarantees appropriate operation for both $\mathrm{X} 2$ at $105 \mathrm{GHz}$ near the main ports with $B_{\max }(0)=1.768 \mathrm{~T}$ and X3 at $140 \mathrm{GHz}$ near the $\mathrm{N}$-ports with $B_{\min }(0)=1.663 \mathrm{~T}$.

For both directions, which are very oblique with $N_{\|} \gtrsim$ 0.4 and $N_{\|} \gtrsim 0.5$, respectively, the condition for cyclotron absorption, $n \omega_{c e} / \omega \geq\left(1-N_{\|}^{2}\right)^{1 / 2}$, is satisfied for $140 \mathrm{GHz}$ and $n=3$ practically for more than half the first ray path inside the plasma (see Fig. 1, bottom). Since the rate of absorption for $\mathrm{X} 3$ is roughly one order magnitude weaker than for X2-mode, the width of the deposition profile is very broad (compare the length about $1 \mathrm{~m}$ with few $\mathrm{cm}$ for $\mathrm{X} 2$ ). Due to the weak $|\nabla B|$ along the ray, the normalized energy of resonant electrons responsible for the absorption varies only slightly along the ray trajectory, $E_{\mathrm{res}} / T_{e} \sim 4$. The most important difference between the two directions is the location of the resonance line in momentum space. For " +15 deg", only the electrons with $u_{\|}<0$ are involved in the resonance, while for " $0 \mathrm{deg}$ " the resonance line occupies both half-planes, $u_{\|} \leq 0$ and $u_{\|} \geq 0$. By steering of $\mathrm{X} 3$ beams by RSL and redirecting significant part of power (from 5\% to $28 \%$ of $6 \mathrm{MW}$ ) to the different fractions of electrons, locally trapped or passing, kinetic effects in radial transport can be studied.

\section{Partial replacement of LFS X2 by HFS X3 in "high-mirror" configuration}

The second example of the selective heating of trapped electrons is based on partial replacement of the X2mode launched near the maximum of $B$ by the X3 mode launched near the minimum of $B$ in the magnetic configuration with high mirror-ratio, $B_{01} / B_{00}=0.246$ and $B_{\max } / B_{\min }=1.64$. This scenario is more attractive than a use of the "standard" configuration since the resonance conditions with the same frequency, $140 \mathrm{GHz}$, can be 

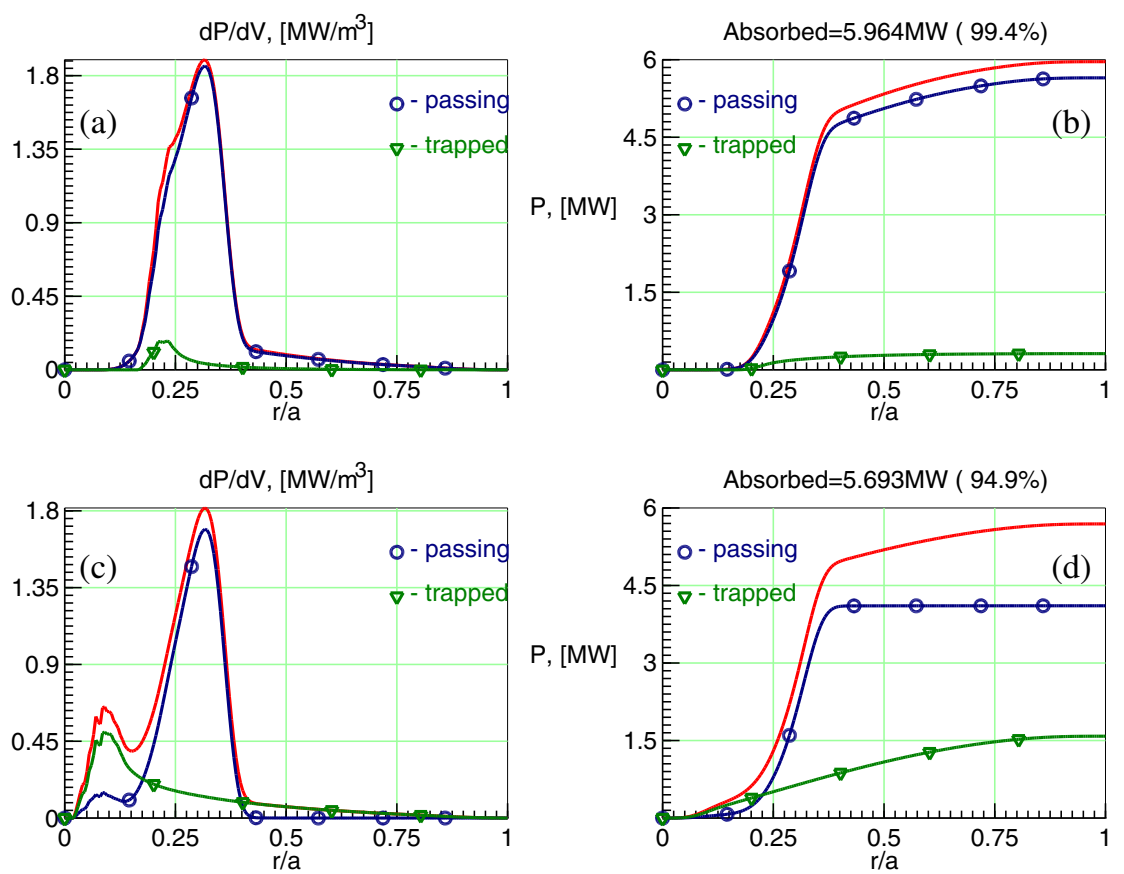

Figure 3. Total deposition profiles (see (a) and (c)) and its integrals (see (b) and (d)) for all RF beams with $P_{R F}^{\text {total }}=6 \mathrm{MW}$ are shown. (a-b): the case " +15 deg" with small heating of trapped electrons, $15 \%$ of the X3 power and $P_{\mathrm{abs}}^{\text {trap }} / P_{\mathrm{abs}}^{\mathrm{total}} \simeq 0.05$. (c-d): the case " 0 deg" with significant heating of trapped electrons, 94\% of X3-mode power and $P_{\mathrm{abs}}^{\text {trap }} / P_{\mathrm{abs}}^{\mathrm{total}} \simeq 0.28$.

satisfied near the maximum and minimum of $B$ for both the $\mathrm{X} 2$ and $\mathrm{X} 3$ modes, respectively, and no reduction of the magnetic field is required. In this configuration, however, dependence of single-pass absorption from the launch-angle is more critical than for the case considered above, and only one angle for injection can be applied, "+15 deg", for which single-pass absorption is approximately $92 \%$ with negligible fraction of power absorbed by passing electrons. For this scenario, the ray trajectories are not shown in the figure, but qualitatively they are similar to those shown in Fig. 1 with only the difference that the absorption for X2 is LFS shifted.

For the pure X2 scenario, five beams $1 \mathrm{MW}$ each are assumed to be launched through the main ports. Two of them are co- and three are counter-directed with respect to the toroidal field that guaranties a compensation of the bootstrap current. In order to switch from pure X2 to the combined X2 \& X3 scenario, two beams, one co- and one counter-directed, have to be replaced by two beams launched from the N-ports. In this case, the total current drive as well as its profile are not changed much and disturbance of the $\iota$-profile and of magnetic configuration is minimized.

Simulations were performed for the density $n_{e}(0)=$ $0.75 \times 10^{20} \mathrm{~m}^{-3}$, Fig. 4 (left), with the aforementioned transport model. The equilibrium was calculated for parabolic $\beta$-profile with $\beta=0.02$. The temperature profiles (see Fig. 4, middle) are flat in the center with $T_{e}(0) \simeq$ $2.5 \mathrm{keV}$ and $T_{i}(0) \simeq 2.0 \mathrm{keV}$. From Fig. 4 one can see also that near $\rho \simeq 0.3$, where the peak of $\mathrm{X} 2$ deposition profile is localed (see Fig. 5), the radial electric field is positive indicating the "electron root" in confinement. Due to a several factors (somewhat higher density and lower power, and, additionally, the different transport properties of the chosen magnetic configuration), the electron temperature is approximately half of the values obtained in the scenario described in previous section.

The oblique LFS X2-mode with $N_{\|} \simeq 0.25$ produces well localized off-axis heating with the deposition profile in $0.15<\rho<0.4$ (see Fig. 5 (a-b)) with the maximum of $d P_{\text {abs }}^{X 2} / d V$ located near $\rho \simeq 0.3$. Plasma is optically thick for $\mathrm{X} 2$ and the power is absorbed near the maximum of $B$ only by passing electrons, i.e. $P_{\text {abs }}^{\text {trap }}=0$, with the main energy of the resonance electrons at about $E / T_{e} \sim 4$. This case, with $5 \mathrm{MW}$ of pure LFS X2 heating, is quite typical for ECRH scenarios and no significant deviation from the transport predictions is expected.

The case of combined LFS X2 (3 MW) and HFS X3 ( $2 \mathrm{MW}$ ) is more complex. Again, plasma for X3 mode is optically gray and very broad deposition profile appears. Similar to above considered scenario, HFS launch of X3 is very oblique with $N_{\|} \gtrsim 0.5$, the condition for cyclotron absorption, $n \omega_{c e} / \omega \geq\left(1-N_{\|}^{2}\right)^{1 / 2}$, is satisfied for $140 \mathrm{GHz}$ and $n=3$ practically from the entrance of the beam in plasma. The normalized energy of resonant electrons varies only slightly along the ray trajectory, $E_{\mathrm{res}} / T_{e} \sim 4$. As it is shown in Fig. 5 (c-d), approximately half of the power from the X3 mode (i.e. $1 \mathrm{MW}$ that is $20 \%$ of the total power) is absorbed strongly off-axis, $\rho>0.4$, while the rest of power from X3 is absorbed in the central domain, making the peaked deposition profile. The most important is that due to specific $B$-dependence along the sight-line, only trapped electrons are responsible for absorption of X3 mode and $P_{\text {abs }}^{\text {trap }} / P_{\text {abs }}^{\text {total }} \simeq 40 \%$. 

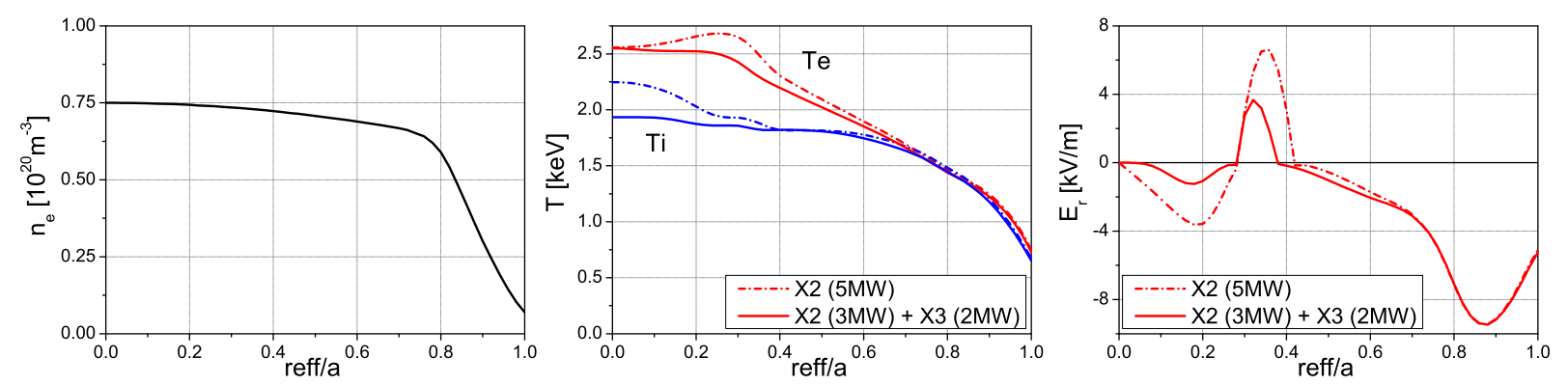

Figure 4. Plasma profiles established for pure LFS X2 (dot-dashed lines) and combined LFS X2 + HFS X3 (solid lines) heating with $P_{R F}^{\text {total }}=5 \mathrm{MW}$ in "high-mirror" magnetic configuration are shown. Left: density (fixed in simulations, assumed to be controlled). Middle: electron and ion temperature profiles for both cases, pure LFS X2 and combined LFS X2 + HFS X3 heating. Right: radial electric field for both cases.

\section{Summary}

In this paper, preliminary results of numerical simulations of ECRH scenarios in the W7-X stellarator with selective heating of trapped/passing electrons by the X3-mode $(140 \mathrm{GHz})$ launched from HFS near the "triangular" plane (minimum of $B$ ) are presented. Two scenarios were considered: the first one is the operation with "standard" magnetic configuration and reduced magnetic field, where the HFS X3-heating at $140 \mathrm{GHz}$ is supposed to be combined with LFS X2-heating at $105 \mathrm{GHz}$, and the second scenario is operation with "high-mirror" configuration where both LFS X2 as well as HFS X3 can be applied with the same frequency $140 \mathrm{GHz}$. Calculations were performed by coupled transport and ray-tracing codes.

For the first scenario it was shown that the power fraction absorbed by trapped electrons from HFS X3 is very sensitive to the launch conditions. In particular, changing the launch angle for the X3 beam from " $0 \mathrm{deg}$ " to "+15 deg", $P_{\text {abs }}^{\text {trap }}$ is increasing from $15 \%$ to $94 \%$ from the power of X3 beam (here, " 0 deg" marks the central line of RSL that corresponds to $N_{\|} \simeq 0.6$ near the axis and " +15 deg" to $N_{\|} \simeq 0.4$ ). For the second scenario, it was shown that partial replacement of LFS X2 heating by HFS X3 produces significant redistribution of the absorbed power between the different fraction of electrons while the plasma profiles are only weakly affected. In particular, $P_{\text {abs }}^{\text {trap }}$ for the considered case is changing from zero to $40 \%$ of $P_{\text {abs }}^{\text {total }}$, i.e. $2 \mathrm{MW}$ from $5 \mathrm{MW}$ of total injected power can be rapidly redirected from the passing to locally trapped electrons of the same energy range.

The power densities shown in Fig. 3 as well in Fig. 5 were calculated under the assumption that the absorbed power is spread over the entire flux surface. However, this is strictly true only for passing electrons. In contrast, the power density calculated by the code for the trapped electrons does not take into account the geometrical factor related to the reduced volume occupied by trapped electrons that are locked within toroidal ripples. As a consequence, the local power density in the cyclotron interaction can be increased significantly, and quasilinear effects may play a non-negligible role. Such effects need to be studied with help of more sophisticated kinetic modeling.
In discussion of possible rapid changes in the $\mathrm{X} 3$ mode absorption, the characteristic times for reaction of the magnetic configuration need to be estimated. With changing the direction of HFS X3 or partial replacement of LFS X 2 by HFS X3 heating, the deposition and plasma profiles are re-establishing within the energy confinement time, $\tau_{E} \simeq 0.25 \mathrm{sec}$. The magnetic equilibrium adopts the finite- $\beta$ effects also with this characteristic time. The characteristic time for establishing the plasma current profile (bootstrap current and ECCD with the inductive screening current) is defined by the diffusive skin-time, $\tau_{J} \simeq 4 \mathrm{sec}$. This means that the equilibrium adopts the new $\iota$-profile much slowly than the $\beta$-profile. Since the plasma profiles are only weakly affected by changing the launch conditions or replecement of the modes, the optimal period for modulation via RSL without significant disturbance of the magnetic equilibria can be chosen from the condition $\tau_{E} \lesssim \Delta t \ll \tau_{J}$. Taking into account that $\tau_{E}$ and $\tau_{J}$ differ of only one order, this condition can be satisfied only marginally if the time period is chosen about $0.3-1 \mathrm{sec}$. However, this time seems to be reasonable for studying in W7-X experiments the kinetic effects specific for stellarators which are not included in the standard neoclassical transport theory.

\section{References}

[1] C. Beidler, G. Grieger, F. Herrnegger, E. Harmeyer, J. Kisslinger, W. Lotz, H. Maassberg, P. Merkel, J. Nuhrenberg, F. Rau et al., Fusion Technol. 17, 148 (1990)

[2] G. Grieger, W. Lotz, P. Merkel, J. Nührenberg, J. Sapper, E. Strumberger, H. Wobig, R. Burhenn, V. Erckmann, U. Gasparino et al., Phys. Fluids B 4, 2081 (1992)

[3] V. Erckmann, P. Brand, H. Braune, G. Dammertz, G. Gantenbein, W. Kasparek, H.P. Laqua, H. Maassberg, N.B. Marushchenko, G. Michel et al., Fusion Sci. Technol. 52, 291 (2007)

[4] G. Dammertz, S. Alberti, A. Arnold, D. Bariou, P. Brand, H. Braune, V. Erckmann, O. Dumbrajs, G. Gantenbein, E. Giguet et al., IEEE Trans. Plasma Sci. 34, 173 (2006) 

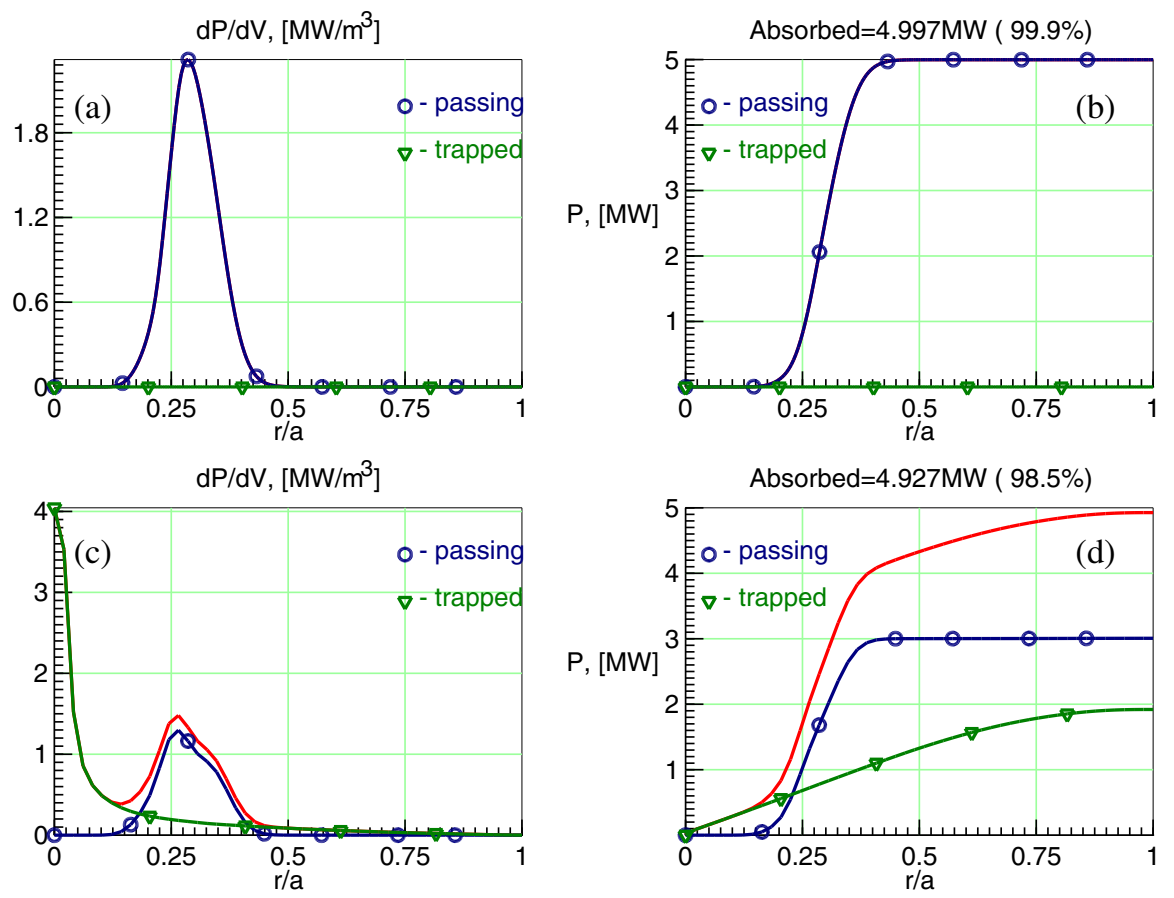

Figure 5. Total deposition profiles (see (a) and (c)) and its integrals (see (b) and (d)) for five RF beams with $P_{R F}^{\text {total }}=5 \mathrm{MW}$ are shown. $(\mathrm{a}-\mathrm{b})$ : pure LFS X2 heating near maximum of $B$ with absorption of only by passing electrons and $P_{\mathrm{abs}}^{\text {trap }} / P_{\mathrm{abs}}^{\text {total }}=0$. (c-d): combined scenario with LFS X2 (3 MW near maximum of $B$ ) and HFS X3 (2 MW near minimum of $B$ ) with significant heating of trapped electrons, $94 \%$ of X3-mode power and $P_{\text {abs }}^{\text {trap }} / P_{\text {abs }}^{\text {total }} \simeq 0.4$.

[5] B. Plaum, V. Erckmann, W. Kasparek, H. Laqua, C. Lechte, M. Weissgerber, Design of a remote steering ECRH launcher for the Stellerator Wendelstein 7-X, in Infrared Millimeter and Terahertz Waves (IRMMW-THz), 2010 35th International Conference on (2010), pp. 1-2

[6] W. Kasparek, C. Lechte, B. Plaum, A. Zeitler, V. Erckmann, H. Laqua, M. Weißgerber, A. Bechtold, M. Busch, B. Szcepaniak, Remote-Steering Launchers for the ECRH system on the Stellarator W7-X, in Proc. 18th Joint Workshop on ECE and ECRH, Nara, Japan, April 2014

[7] V. Erckmann, U. Gasparino, Plasma Phys. Control. Fusion 36, 1869 (1994)

[8] M. Romé, V. Erckmann, U. Gasparino, H.J. Hartfuß, G. Kühner, H. Maaßberg, N. Marushchenko, Plasma Phys. Control. Fusion 39, 117 (1997)

[9] H. Maassberg, C.D. Beidler, U. Gasparino, M. Romé, K.S. Dyabilin, N.B. Marushchenko, S. Murakami, Phys. Plasmas 7, 295 (2000)

[10] M. Romé, C.D. Beidler, H. Maßßberg, N.B. Marushchenko, Y.A. Turkin, the W7-AS Team, Plasma Phys. Control. Fusion 48, 353 (2006)

[11] M. Hirsch, J. Baldzuhn, C. Beidler, R. Brakel, R. Burhenn, A. Dinklage, H. Ehmler, M. Endler,
V. Erckmann, Y. Feng et al., Plasma Phys. Control. Fusion 50, 053001 (2008)

[12] S. Murakami, U. Gasparino, H. Maaßberg, N. Marushchenko, N. Nakajima, M. Okamoto, M. Romé, the W7-AS Team, J. Plasma Fusion Res. Ser. 1, 122 (1998)

[13] J. Seol, C.C. Hegna, J.D. Callen, Phys. Plasmas 14, 082505 ( 8) (2007)

[14] R. Kamendje, S.V. Kasilov, W. Kernbichler, M.F. Heyn, Phys. Plasmas 10, 75 (2003)

[15] N. Marushchenko, Y. Turkin, H. Maassberg, Comp. Phys. Comm. 185, 165 (2014)

[16] Y. Turkin, C.D. Beidler, H. Maaßberg, S. Murakami, V. Tribaldos, A. Wakasa, Phys. Plasmas 18, 022505 (2011)

[17] S.P. Hirshman, J.C. Whitson, Phys. Fluids 26, 3553 (1983)

[18] S.P. Hirshman, K.S. Chaing, W.I. van Rij, J.C.O. Beasley, E.S. Crume, Phys. Fluids 29, 2951 (1986)

[19] V. Erckmann, H.P. Laqua, H. Maassberg, J. Geiger, G. Dammertz, W. Kasparek, M. Thumm, W7$\mathrm{X}$ and W7-AS teams, I. and W7-X team, FZK Karlsruhe W7-X team et al., Fusion Eng. Design 53, 365 (2001) 